\title{
Optimal Hard Decision Fusion Rule for Centralized and Decentralized Cooperative Spectrum Sensing in Cognitive Radio Networks
}

\author{
Ashish Rauniyar, Jae Min Jang, and Soo Young Shin
}

\begin{abstract}
Cognitive radio (CR) is the key technology for unlicensed secondary users (SUs) to exploit the unused spectrum of primary users (PUs) via opportunistic spectrum access. In CR networks, SUs can be coordinated to perform cooperative spectrum sensing (CSS) to achieve higher detection accuracy. The fusion center in centralized CSS collects all the individual local spectrum sensing information from the respective SUs and combines the local spectrum sensing result by applying appropriate fusion rule to give a final collaborative decision whether to proceed with spectrum access or not. Basically, there exist two kinds of detection errors (i.e., miss-detection error and false-alarm error) in spectrum sensing, which degrade the sensing performance severely. Aiming to minimize the probability of total detection errors, we first dissect different hard decision fusion rule and we establish that "Half-Voting (HV)" rule is the optimal fusion rule applied to the detector for centralized CSS. Next, the performance of different hard decision fusion rule in AWGN channel and Rayleigh fading channel is carried out in MATLAB to find out the optimal hard decision fusion rule in centralized and decentralized CSS.
\end{abstract}

Index Terms-Cognitive radio, centralized, decentralized, hard decision fusion, optimization, Rayleigh fading.

\section{INTRODUCTION}

COGNITIVE radio (CR) enables opportunistic access to unused licensed bands. CR allows secondary users (SUs) to utilize the free portions of licensed spectrum while ensuring no interference to primary users (PUs) transmissions [1], [2]. In a recent survey conducted by the Federal Communications Commission (FCC) on spectrum utilization has indicated that the actual licensed spectrum is largely under-utilized in vast geographical dimensions [3]. SU first sense the activities of PU and access the spectrum holes (white spaces), if no primary activities are detected. Sensing accuracy is important for avoiding interference to the PUs in CR technology. Reliable spectrum sensing is not always guaranteed, due to the multipath fading, shadowing and hidden terminal problem. Single node spectrum sensing detection performance is

Manuscript received October 3, 2014; revised May 25, 2015. This work was supported by MSIP (Ministry of Science, ICT and Future Planning), Korea, under the "Creative ICT Convergence Human Resource Development Program" support program and "Global IT Talent" support program (NIPA-2014-H0904-14-1005) supervised by the NIPA (National IT Industry Promotion Agency).

Ashish Rauniyar and Jae Min Jang are with the Department of IT Convergence Engineering, Kumoh National Institute of Technology, South Korea (e-mail: ashish.rauniyar@kumoh.ac.kr, oops_kichin@ naver.com).

Soo Young Shin is with the School of Electronics Engineering, Kumoh National Institute of Technology, South Korea (e-mail: wdragon@kumoh.ac.kr). deteriorated due to these factors. Cooperative spectrum sensing (CSS) has thus been widely studied for quick and reliable detection [4]-[6].

Cooperative spectrum sensing can be done either in the centralized or decentralized approach. Centralized CSS has two successive stages, sensing and reporting. In sensing stage, spectrum sensing is done by several local secondary users. Then in next stage, PU sensing measurements are sent to fusion center (FC) to combine them and make a better overall decision by applying one of the different hard decision fusion rules. In decentralized CSS approach, there is no fusion center dedicated is assumed. At first, each CR users will perform the local spectrum sensing individually. Next, each of the individual sensing information will be exchanged among the collaborating users to decide on the collective probability of PU using appropriate hard decision fusion rule. Further, in spectrum sensing, there exist two kinds of detection errors i.e., miss-detection error and false-alarm error, which degrade the sensing performance severely. There are several kinds of decision fusion rules in centralized and decentralized CSS such as soft decision fusion [7] and hard decision fusion rule [8] to finalize on a collaborative decision.

In CSS, sensing should be done faster by the SU, so that more time can be assigned for data transmission in a fixed frame format, which will increase the throughput of the system [9]. Since, soft decision fusion rule has to process several bits of sensing information for the sensing decision; there can be a delay in sensing with soft decision fusion rule. In the other hand, only one bit of information is required as " $1=\mathrm{PU}$ is present" or " $0=\mathrm{PU}$ is absent" in hard decision fusion rule to finalize on a collaborative decision by applying appropriate hard decision fusion rule. As, only 1 bit of information is processed for spectrum sensing decision by applying hard decision fusion rule, more time can be assigned for the data transmission which will increase the throughput of the system. The quick question arises here, which hard decision fusion rule should be applied for the CSS for the optimal performance. Most of the works are carried out on centralized CSS such as in [4], [5], the authors have only considered a single hard decision fusion rule at the FC for the data fusion. Still, we are not sure of choosing the right hard decision fusion rule at the FC for the optimal sensing performance of the detector. Moreover, there is very less significant research works concentrated on decentralized CSS. We are also not certain of choosing the appropriate hard decision fusion rule for the decentralized CSS approach. Therefore, in this paper, our main focus is on the study of different hard decision fusion rule and their performance in 
centralized and decentralized CSS in AWGN and Rayleigh fading channel to get the optimal hard decision fusion rule for the CSS.

There are several spectrum sensing techniques available as of now. Among several spectrum sensing techniques such as the matched filter detection and the cyclostationary feature detection; energy detection (ED) is the most popular method employed for spectrum sensing. Measuring only received signal power; the energy detector is a non-coherent detection device with low implementation complexity. All these spectrum sensing techniques are well documented in the literature [10]. Throughout this paper, we will focus on ED because of its ease of implementation.

In this paper, we first analyze the different hard decision fusion rule with energy detection to minimize the total error rate in centralized CSS. In particular, through our simulation result, we will show that that "Half Voting" or "Majority" rule is the most optimal hard decision fusion rule compared to other hard decision fusion rules. The performance of different hard decision fusion rule for centralized and decentralized CSS in AWGN and Rayleigh fading channel is also carried out in MATLAB in terms of detection performance to find out the overall optimal hard decision fusion rule.

The rest of the paper is organized as follows. In Section II, system model, preliminaries of energy detection, centralized and decentralized CSS is briefly introduced. Section III is devoted to the different hard decision fusion rule. Simulation result is presented in Section IV and finally conclusion is drawn in Section V.

\section{SYSTEM MODEL}

The main goal of CR is to correctly identify the presence and absence of PU and allows the SUs to utilize the unused spectrum, if it is not used by licensed PUs. Under binary hypothesis testing, we consider the occurrence of two input events in observing signal $x_{i}$ in some observation interval denoted by [11].

$$
\begin{aligned}
& H_{0}: x_{i}=n_{i} \\
& H_{1}: x_{i}=h_{i} \times s_{i}+n_{i}
\end{aligned}
$$

where $i=1,2,3, \ldots, N$ is the number of samples and $h_{i}$ is the channel response. $H_{0}$ represents the hypothesis that the observation vector consists of noise. $H_{1}$ represents the hypothesis that the observation vector consists of noise and signal. The noise component $n_{i}$ is assumed to be Additive White Gaussian random variable which is independent and identically-distributed (i.i.d) with zero mean normal distribution with variance $\sigma^{2} \sim N\left(0, \sigma^{2}\right)$ and $s_{i}$ is the signal.

\section{A. Energy Detector (ED)}

The ED is non-coherent detector which detects the presence of signals by simply squaring its energy and comparing that energy around the carrier frequency with certain threshold. The ED consists of a quadrature receiver with $y_{I}$ and $y_{Q}$ representing samples from In-phase and Quadrature branch respectively. The samples after passing the squaring device, output of the integrator is denoted by [12].

$$
y_{I}=y_{Q}=\left(\frac{1}{N_{0}}\right) \int_{0}^{T} r^{2}(t) d t
$$

where $r(t)$ is input signal, $N_{0}$ is noise spectral density.

Within observed sensing period, test statistic of ED can be approximated as $Y_{E D}=y_{I}+y_{Q}$. At the observation time $t$, decision variable $Y_{E D}$ will be compared to a detection threshold of ED denoted by $\lambda_{E D}$. Threshold value is set to meet the target probability of false alarm $p_{f a}$ according to the noise power. In the case of $p_{f a}$, the detector classifies the channel as busy when the actual channel is free. Probability of detection $\left(p_{d}\right)$ is the probability of correctly detecting the presence of PU when the actual channel is in busy state. The expression for $p_{f a}$ and $p_{d}$ can be given as [13].

$$
p_{f a}^{E D}=1-F_{x}\left(\frac{\lambda_{E D}}{\sigma^{2}}, 2 n\right),
$$

where $F_{x}$ is cumulative distribution function (CDF) of standard chi-square random variable with $k$ degree of freedom.

$$
p_{d}^{E D}=Q_{u}\left(\sqrt{n(S N R)}, \quad \sqrt{\frac{\lambda_{E D}}{\sigma^{2}}}\right)
$$

where $Q$ is generalized Marcum- $Q$ function, $u$ is the time-bandwidth factor.

Probability of miss detection $\left(p_{m}\right)$ can also be identified in ED. $p_{m}$ is the probability that the ED can miss to detect the presence of PU. $p_{m}$ can be given as [13].

$$
p_{m}^{E D}=1-p_{d}^{E D}
$$

Generally, there exist two kinds of detection errors i.e., miss-detection error $p_{m}$ and false-alarm error $p_{f a}$, which degrade the sensing performance of ED critically [14]. If the total error rate $p_{e}$, is the sum of the probability of false alarm $p_{f a}$ and the probability of missed detection $p_{m}$. Thus, the total error rate is given by

$$
p_{e}^{E D}=p_{f a}^{E D}+p_{m}^{E D}
$$

When the composite received signal consists of a large number of plane waves, for some types of scattering environments, the received signal has a Rayleigh distribution. If the signal amplitude follows a Rayleigh distribution, then the SNR follows an exponential probability density function (PDF) which is given by [15].

$$
f(\gamma)=\frac{1}{\gamma} \exp \left(-\frac{\gamma}{\gamma}\right), \gamma \geq 0
$$

In this case, a closed-form formula for probability of detection for energy detector in Rayleigh fading channel can be given as [15]. 


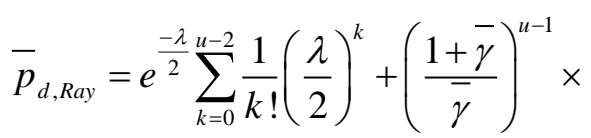

$$
\begin{aligned}
& \left(e^{-\frac{\lambda}{2(1+\bar{\gamma})}}-e^{-\frac{\lambda}{2} \sum_{k=0}^{u=2} \frac{1}{k !}\left(\frac{\lambda \bar{\lambda}}{2(1+\bar{\gamma}}\right)}\right)
\end{aligned}
$$

\section{B. Centralized Cooperative Spectrum Sensing (CCSS)}

Reliable spectrum sensing is not always guaranteed, due to multipath fading, shadowing and hidden terminal problem. Single node spectrum sensing detection performance is deteriorated due to these reasons. Thus, the detection probability can be improved by employing multiple cognitive radios which will work in cooperation and minimize the overall total error rate.

Fig. 1 shows the arrangement of centralized cooperative spectrum sensing in which multiple SUs collaborate with each other and send their local sensing information to the FC or base station. All the local sensing information from individual SU will be processed at fusion center with one of the hard decision fusion rule and finally an overall decision will be given by fusion center, whether to go with spectrum access or not [16].

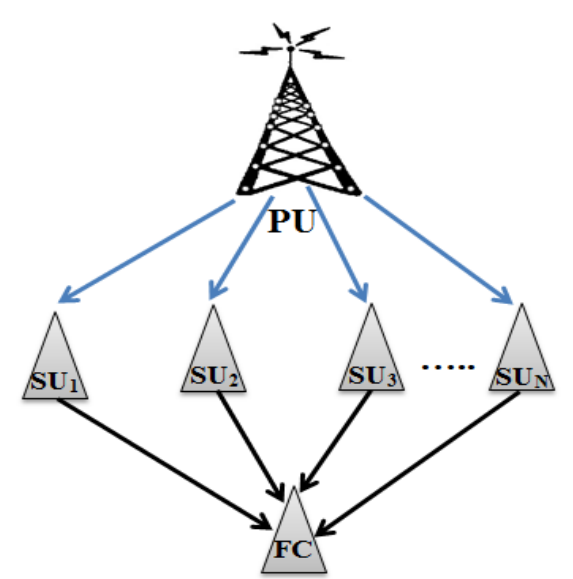

Fig. 1. Centralized cooperative spectrum sensing.

\section{Decentralized Cooperative Spectrum Sensing (DCSS)}

As there is no fusion center dedicated is assumed in DCSS, each SU users will perform the local spectrum sensing individually. The individual sensing information will be exchanged among the collaborating users to decide on the presence or absence of PU in a collaborative way. Each SU will make their final decision based on the sensing information it received from other SUs using appropriate hard decision fusion rule [17].

Fig. 2 shows the arrangement of DCSS where there are two cooperative groups of SUs consisting of $3 \mathrm{SU}$ in each group. Each SU user will detect the frequency channel of PU whether it is occupied or not, in their own group. Then it will compare with SU users in their group by using appropriate hard decision fusion rule. Subsequently, it will compare their group decision with other group decision by using hard decision fusion rule again to decide the presence or absence of PU. As we can clearly see from Fig. 2, the hard decision fusion rules are used twice for DCSS approach i.e., first hard decision fusion in comparing the decision among themselves in one group and the second hard decision fusion in comparing the final decision of the two groups. Therefore, the different hard decision fusion rule in this approach can be treated as "OR-OR", "AND-AND", "HV-HV” rule.

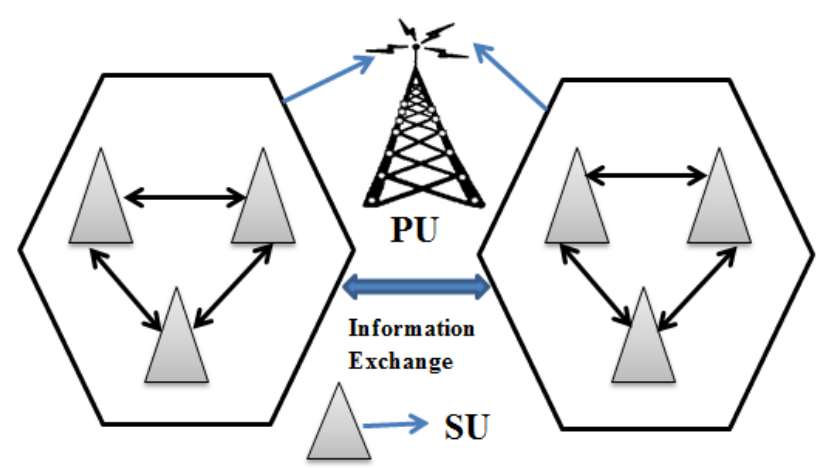

Fig. 2. Decentralized cooperative spectrum sensing.

\section{HARD DECISION FUSION RULE}

Each CR user makes its own decision regarding the presence and absence of PU and forwards the one bit binary decision (1 or 0 ) to FC or cooperative groups for data fusion. We assume that the PU is located far away from all SUs. All the SUs receive the primary signal with same local mean signal power, i.e. all SUs form a cluster with distance between any two SUs is negligible compared to the distance from the PU to a SU. For simplicity, we have also assumed that the noise, fading statistics and average SNR are the same for each SU user.

As, only 1 bit of information is processed for spectrum sensing decision to the FC or cooperative groups, more time can be assigned for the data transmission in a fixed frame which will increase the throughput of the system unlike soft decision fusion rule where lots of bits information are forwarded for decision fusion. The cooperative probability of detection $Q_{d}$ at the fusion center is given by [14].

$$
Q_{d}^{E D}=\sum_{l=n}^{K}\left(\begin{array}{c}
K \\
l
\end{array}\right) p_{d, i}^{l}\left(1-p_{d, i}^{K-l}\right)
$$

where $K$ is the total number of secondary users $\& 1<n<K$.

Similarly, cooperative probability of false alarm $Q_{f a}$ and cooperative miss-detection probability $Q_{m}$ at the fusion center is given by [14].

$$
\begin{gathered}
Q_{f a}^{E D}=\sum_{l=n}^{K}\left(\begin{array}{c}
K \\
l
\end{array}\right) p_{\mathrm{fa}, i}^{l}\left(1-p_{\mathrm{fa}, i}^{K-l}\right) \\
Q_{m}^{E D}=1-Q_{d}^{E D}
\end{gathered}
$$

There are different types of hard decision fusion rule for the cooperative spectrum sensing. They are explained below.

\section{A. OR Rule}

In OR fusion rule, if any one of the local decisions sent to the $\mathrm{FC}$ is a logical one i.e., decision $H_{1}$, the final decision 
made by the FC using this rule is one i.e., decision $H_{1}$. The evaluation of the OR hard decision fusion rule can be carried out by setting $K=1$ in Eq. (9) and (10). The $Q_{d}$ and $Q_{f a}$ at the $\mathrm{FC}$ using OR rule is given by

$$
\begin{gathered}
Q_{d, O R}^{E D}=1-\left(1-p_{d, i}^{K}\right) \\
Q_{\mathrm{fa}, O R}^{E D}=1-\left(1-p_{f a, i}^{K}\right)
\end{gathered}
$$

\section{B. AND Rule}

In AND fusion rule, if all of the local decisions sent to the FC by all SUs is a logical one i.e., decision $H_{1}$, the final decision made by the FC using this rule is one i.e., decision $H_{1}$. The evaluation of the AND hard decision fusion rule can be carried out by setting $l=K$ in Eq. (9) and (10). The $Q_{d}$ and $Q_{f a}$ at the FC using AND rule is given by

$$
\begin{gathered}
Q_{\mathrm{d}, \mathrm{AND}}^{E D}=p_{d, i}^{K} \\
Q_{\mathrm{fa}, \mathrm{AND}}^{E D}=p_{\mathrm{fa}, i}^{K}
\end{gathered}
$$

\section{Half Voting (HV) Rule or Majority Rule}

In this fusion rule, if the local decisions sent to the fusion center by at least half of SUs is a logical one i.e., decision $H_{1}$, the final decision made by the $\mathrm{FC}$ using this rule is one i.e., decision $H_{1}$. Here, the final decision follows the majority of the local decision of the SUs. The evaluation of this hard decision fusion rule can be carried out by setting $l=n / 2$ in Eq. (9) and (10). The $Q_{d}$ and $Q_{f a}$ at the FC using $\mathrm{HV}$ rule is given by:

$$
\begin{gathered}
Q_{d, H V}^{E D}=\sum_{l=n / 2}^{K}\left(\begin{array}{c}
K \\
l
\end{array}\right) p_{d, i}^{l}\left(1-p_{d, i}^{K-l}\right) \\
Q_{\mathrm{fa}, H V}^{E D}=\sum_{l=n / 2}^{K}\left(\begin{array}{c}
K \\
l
\end{array}\right) p_{\mathrm{fa}, i}^{l}\left(1-p_{\mathrm{fa}, i}^{K-l}\right)
\end{gathered}
$$

\section{Simulation Results}

Fig. 3 shows the simulation result for different fusion rules from $n=1$ to $K=6$ in a cognitive radio network with 6 SUs in terms of total error rate $p_{e}=Q_{f a}^{E D}+Q_{m}^{E D}$ verses threshold of ED. A $S N R$ of $10 \mathrm{~dB}$ and time bandwidth factor $u=10$ and AWGN channel was considered for simulation. From the Fig. 3 , we can verify that, for a fixed small threshold, the optimal hard decision fusion rule is the "AND" rule, i.e. $K=6$ and for a fixed large threshold, the optimal fusion rule is the "OR" rule, i.e. $n=1$. Finally, we can see that the optimal hard decision fusion rule is "Half voting" or "Majority" rule i.e. $n=$ 3 over all range of detection threshold in optimizing the total detection errors in centralized cooperative spectrum sensing. We further confirmed this by modelling our system for $n=3$ using energy detector.
Fig. 4 shows the receiver operating characteristic (ROCs) curve for energy detector using hard decision fusion rule in CCSS. A SNR of $6 \mathrm{~dB}$, time bandwidth factor $u=5$ and AWGN channel was considered for simulation. 6 SUs are cooperating with each other for spectrum sensing. From the Fig. 4, we can see that "OR" rule detection performance is better than the other hard decision fusion rules. Also, it can be seen that the "AND" rule detection performance is lower than "OR" and "HV" hard decision fusion rule.

Fig. 5 shows the CSSS performance under the Rayleigh fading channel for energy detector using different hard decision fusion rule in terms of miss-detection probability. A SNR of $6 \mathrm{~dB}$, time bandwidth factor $u=5$ and Rayleigh fading channel was considered for simulation. 6 SUs are cooperating with each other for spectrum sensing. The simulation for AWGN and Rayleigh fading using $1 \mathrm{SU}$ is also shown for comparison basis. From the Fig. 5, we can see that "OR" hard decision fusion rule miss detection probability is lower than other hard decision fusion rules. Also, it can be seen that the "AND" rule miss-detection performance is higher than "OR" and "HV" hard decision fusion rule at all $p_{f a}$ values.

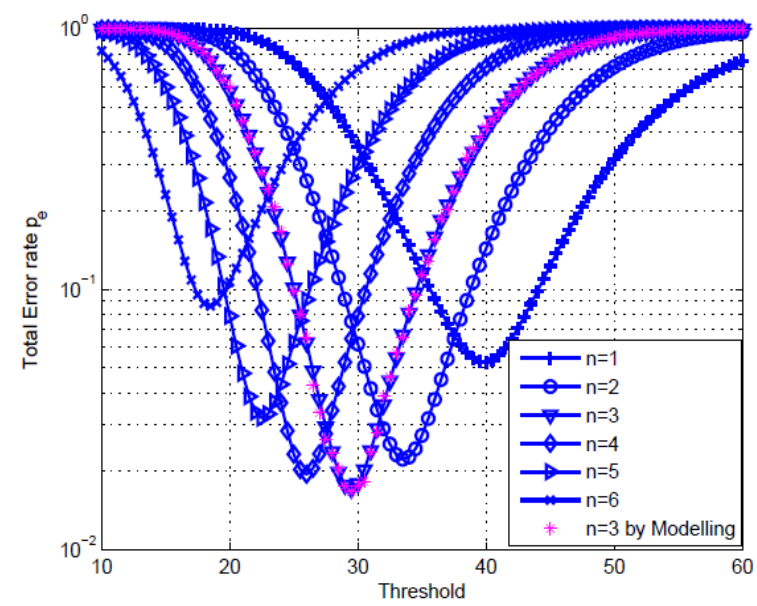

Fig. 3. Total error probability $p_{e} \mathrm{~V} / \mathrm{s}$ detection threshold for various fusion rules when $n=1,2, \ldots, K=6$.

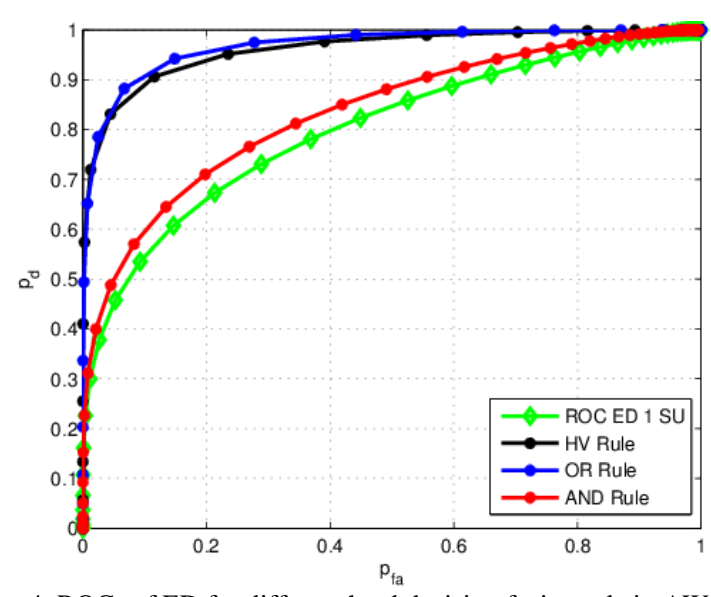

Fig. 4. ROCs of ED for different hard decision fusion rule in AWGN channel.

Fig. 6 shows the DCSS performance under the Rayleigh fading channel for ED using different hard decision fusion rule in terms of detection probability. A $S N R$ of $6 \mathrm{~dB}$, time bandwidth factor $u=5$ and Rayleigh fading channel was 
considered for simulation. The simulation scenario as shown in Fig. 2 was considered, where there are two cooperative groups of SUs consisting of $3 \mathrm{SU}$ in each group. SU will compare their group decision with other group decision by using hard decision fusion rule such as "OR-OR", "AND-AND", "HV-HV". It is clear from the Fig. 6 that the "OR-OR" hard decision fusion rule outperforms the other fusion rules in DCSS in terms of probability of detection of PU. Here also "AND-AND" fusion rule detection performance is lower compared to other hard decision fusion rules.

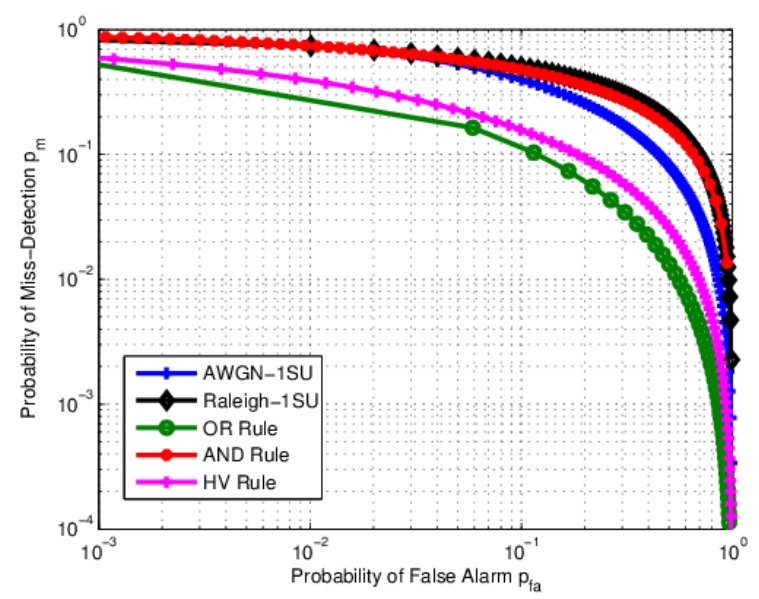

Fig. 5. Centralized cooperative spectrum sensing performance under the Rayleigh fading channel.

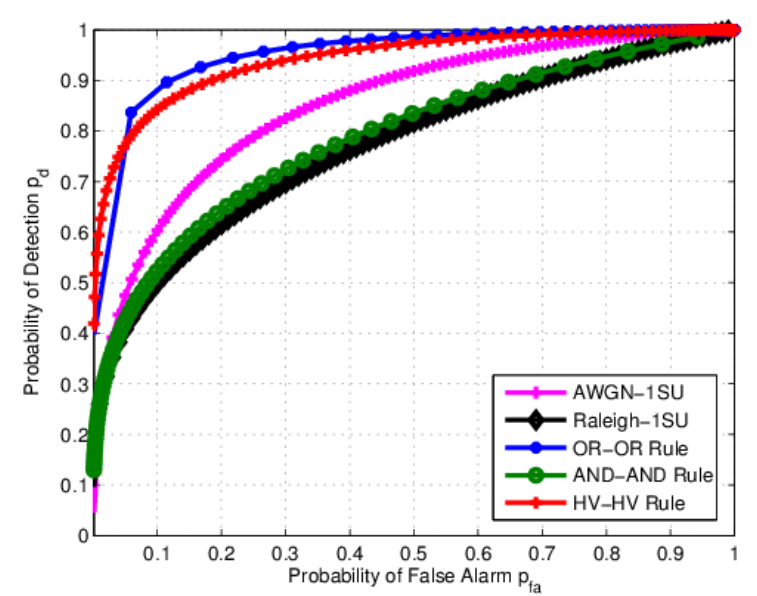

Fig. 6. Decentralized cooperative spectrum sensing performance under the Rayleigh fading channel.

\section{CONCLUSION}

Cooperative spectrum sensing can greatly reduce the false alarm probability and increase the detection probability of the secondary users. To achieve higher detection accuracy of the detector, we must minimize the total error rate i.e. probability of miss detection and probability of false alarm. In order to reduce total error rate, we conclude through our simulation and modeling results ( $n=3$ using energy detector), that the optimal hard decision fusion rule for centralized cooperative spectrum sensing is the "Half Voting" or "Majority" rule. The ROC curve for the AWGN and Rayleigh case provides a comprehensive picture of the detection performance of the centralized and decentralized cooperative spectrum sensing system using different hard decision fusion rule. It is clear from the simulation results that "OR" rule is the optimum hard decision fusion rule compared to "AND" and "HV" rule for both centralized and decentralized cooperative spectrum sensing. "AND" rule shows the worst performance as compared to "OR" rule and "HV" rule. The paper also provided a verification of the validity of the "OR", "AND" and "HV" hard decision fusion schemes under AWGN and Rayleigh fading channel making "OR" rule as optimal choice for hard decision fusion rule in centralized and decentralized cooperative spectrum sensing.

\section{ACKNOWLEDGMENT}

We thank MSIP (Ministry of Science, ICT and Future Planning), Korea, under the "Creative ICT Convergence Human Resource Development Program" support program and "Global IT Talent" support program (NIPA-2014-H0904-14-1005) supervised by the NIPA (National IT Industry Promotion Agency) for providing their support for this work.

\section{REFERENCES}

[1] J. Mitola and G. Q Maguire Jr., "Cognitive radio: Making software radios more personal," IEEE Personal Communications, vol. 6, no. 4, pp. 13-18, 1999.

[2] S. Haykin, "Cognitive radio: Brain-empowered wireless communications," IEEE Journal on Selected Areas in Communications, vol. 23, no. 2, pp. 201-220, 2005.

[3] Federal Communications Commission, "Spectrum policy task force," Report Docket No. 02-135, 2002.

[4] G. Ganesan and Y. Li, "Cooperative spectrum sensing in cognitive radio, part ii: Multiuser networks," IEEE Transactions on Wireless Communications, vol. 6, no. 6, pp. 2214-2222, 2007.

[5] S. M. Mishra, A. Sahai, and R. W. Brodersen, "Cooperative sensing among cognitive radios," IEEE International Conference on Communications, vol. 4, pp. 1658-1663, 2006.

[6] R. Fan and H. Jiang, "Optimal multi-channel cooperative sensing in cognitive radio networks," IEEE Transactions on Wireless Communications, vol. 9, no. 3, pp. 1128-1138, 2010.

[7] H. Uchiyama, K. Umebayashi, T. Fujii, K. Sakaguchi, Y. Kamiya, and Y. Suzuki, "Study on soft decision based cooperative sensing for cognitive radio networks," IEICE Transactions on Communications, vol. 91, no. 1, pp. 95-101, 2008.

[8] J. Shen, S. Liu, R. Zhang, and Y. Liu, "Soft versus hard cooperative energy detection under low SNR," in Proc. IEEE Third International Conference on Communications and Networking in China, 2008, pp. 128-131.

[9] E. Chu, Y. Peh, Y.-C. Liang, Y. L. Guan, and Y. Zeng, "Optimization of cooperative sensing in cognitive radio networks: A sensing-throughput tradeoff view," IEEE Transactions on Vehicular Technology, vol. 58, no. 9, pp. 5294-5299, 2009.

[10] T. Yucek and H. Arslan, "A survey of spectrum sensing algorithms for cognitive radio applications," IEEE Communications Surveys \& Tutorials, vol. 11, no. 1, pp. 116-130, 2009.

[11] J. J. Lehtomaki, J. Vartiainen, R. Vuohtoniemi, and H. Saarnisaari, "Adaptive FCME-based threshold setting for energy detectors," in Proc. the 4th International Conference on Cognitive Radio and Advanced Spectrum Management, 2011, p. 33.

[12] K. Y. Park, "Performance evaluation of energy detectors," IEEE Transactions on Aerospace and Electronic Systems, vol. 2, pp. 237-241, 1978.

[13] D. Triwicaksono and S. Y. Shin, "Energy detector and matched filter as cascaded clear channel assessment in wireless network," in Proc. IET International Conference on Information and Communications Technologies, 2013, pp. 551-556.

[14] W. Zhang, R. K. Mallik, and K. Letaief, "Optimization of cooperative spectrum sensing with energy detection in cognitive radio networks,' IEEE Transactions on Wireless Communications, vol. 8, no. 12, pp 5761-5766, 2009.

[15] M. K. Simon and M.-S. Alouini, Digital Communication Over Fading Channels, John Wiley \& Sons, vol. 95, 2005. 
[16] C. Sun, W. Zhang, and K. Letaief, "Cooperative spectrum sensing for cognitive radios under bandwidth constraints," in Proc. IEEE Wireless Communications and Networking Conference, 2007, pp. 1-5.

[17] N. Noorshams, M. Malboubi, and A Bahai, "Centralized and decentralized cooperative spectrum sensing in cognitive radio networks: A novel approach," in Proc. IEEE Eleventh International Workshop on Signal Processing Advances in Wireless Communications, June 2010, pp. 1-5.

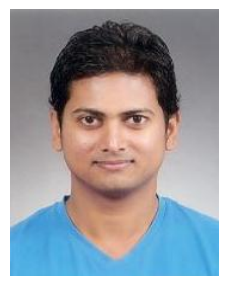

Ashish Rauniyar was born in 1988. He received his undergraduate degree in computer science and technology from University of Mysore, India in 2011. After his undergraduate degree, he worked as a software developer for Wipro Technologies, Bangalore, India from 2011 to 2012. Currently he is working as a graduate research assistant at the Wireless Emerging Networking System Lab, while attending graduate school at Kumoh National Institute of Technology, South Korea. His main research area includes cognitive radio network, MIMO communications, embedded systems and networks, and software engineering.

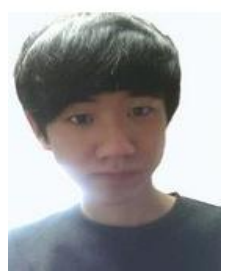

Jae Min Jang was born in 1988. He received his undergraduate degree in electronics engineering from Kumoh National Institute of Technology, South Korea in 2014. Currently he is working as a graduate research assistant at the Wireless Emerging Networking System Lab, while attending graduate school at Kumoh National Institute of Technology, South Korea. His main research area includes MIMO-OFDM, cognitive radio, and mobile application (Android/IOS).

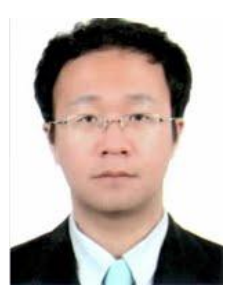

Soo Young Shin was born in 1975 . He received his B.S., M.S., and Ph.D. degrees in electrical engineering and computer science from Seoul National University, Korea in 1999, 2001, and 2006, respectively. He was a visiting scholar in FUNLab at University of Washington, US, from July 2006 to June 2007. After 3 years working in WiMAX Design Lab. of Samsung Electronics, he is now an assistant professor in the School of Electronics in Kumoh National Institute of Technology since September 2010. His research interests include wireless LAN, WPAN, WBAN, wireless mesh network, sensor networks, coexistence among wireless networks, industrial and military network, cognitive radio networks, and next generation mobile wireless broadband networks. 